\title{
Effectiveness of the Vital Aging program to promote active aging in Mexican older adults
}

\author{
This article was published in the following Dove Press journal: \\ Clinical Interventions in Aging \\ 14 November 2016 \\ Number of times this article has been viewed
}

\author{
Neyda Ma Mendoza- \\ Ruvalcabal \\ Rocío Fernández- \\ Ballesteros ${ }^{2}$ \\ 'Health Sciences Department, \\ University of Guadalajara, University \\ Center of Tonalá, Tonalá, Jalisco, \\ Mexico; ${ }^{2}$ Department of Biological \\ and Health Psychology, Autonomous \\ University of Madrid, Madrid, Spain
}

Correspondence: Neyda Ma MendozaRuvalcaba

Health Sciences Department, University of Guadalajara, University Center of Tonalá, CUTONALA, Av. Nuevo Periférico No 555, Ejido San José Tatepozco, CP 48525, Tonalá, Jalisco, Mexico

Tel +52 3335403020 ext 64052 Email nmendoza_ruvalcaba@yahoo. com.mx
Introduction: Aging is not only a population phenomenon but also an experience and an individual reality. Vital Aging ${ }^{\circledR}$ is a program that considers active aging as the lifelong adaptation process of maximizing health and independence, physical and cognitive functioning, positive affect regulation and control, and social engagement. Through its different versions and editions, it has demonstrated being an effective program to promote active aging. The aim of this study is to determine the effectiveness of the "face-to-face" and "combined" versions of the program to promote active aging in Mexican older adults trial.

Methods: Seventy-six older adults aged 60 years and over participated in a quasi-experimental study and were recruited in a senior center to participate in the two experimental conditions: Vital Aging face-to-face (VA-FF) $(n=35)$ and Vital Aging combined (VA-C; multimedia/face-toface) $(n=15)$, and the remaining 26 adults were assigned to a control group. Pretest and posttest assessments were performed after the theoretical-practical intervention. Mean differences and size effects were calculated for estimating the effect of the program.

Results: At the end of the study, participants showed improvements in the active aging outcome measures. Positive effects were observed in the frequency of intellectual, cultural - artistic, and social activities, perceptions of aging, satisfaction with social relationships, and self-efficacy for aging. Additionally, those who participated in VA-FF showed better memory performance, meta-memory, and a trend to report less memory problems, while older persons in VA-C showed a trend to have better life satisfaction. No effects were observed in physical activity, frequency of social relationships, and subjective health.

Conclusion: Findings show that the Vital Aging program in face-to-face and combined versions encourages active aging in Mexican older persons. These results are in general similar to those found in editions performed in Spain, revealing its consistency as a cross-cultural practical initiative for promoting active aging.

Keywords: Vital Aging, active aging, intervention program, successful aging

\section{Introduction}

Population aging is occurring in all the major areas of the world; globally, the share of older people (60 years and older) increased from $9 \%$ in 1994 to $12 \%$ in 2014, and it is expected to reach $21 \%$ by $2050 .^{1}$

There are critically important issues related to the aging society, such as future intergenerational relations and tensions, socioeconomic disparities and inequalities, changes in the structure and function of the family and its capacity to serve the traditional safety-net role, the impact of technology, and the critical importance of adaptation of core societal institutions, including education, work and retirement, housing, transportation, and even the design of the built environment. ${ }^{2}$ However, the main concern in an aging population is the fact that age is highly associated with illness 
and disability. ${ }^{3,4}$ The assumption that increasing numbers of older people, especially very old people, per se mean a parallel rise in social protection costs is erroneous; nonetheless, there is an association between advanced old age and disability and, in turn, with health care costs. Therefore, it is important to implement active aging strategies sensitive to this relationship and aim to recognize and prevent ill health and disability, ${ }^{5}$ not only at policy level but also at individual level, since aging is not only a population phenomenon but also an experience and an individual reality. ${ }^{6}$

The World Health Organization (WHO) proposed a policy framework that considers active aging as a positive "process of optimizing opportunities for health, participation and security in order to enhance quality of life as people age". 7 This concept was further refined with the addition of "lifelong learning" as a fourth component, which implies the opportunity to acquire and update knowledge and skills in order to stay relevant and better assure personal security. Learning is understood as a renewable resource that enhances the capacity to remain healthy; it can occur in formal contexts (to obtain a grade or diploma), nonformal contexts (through planned activities such as workshops, short courses, and seminars), or informal settings (daily life experiences); lifelong learning is important because the access to information is considered a vital key to active aging. ${ }^{8}$ Thus, "active aging" is a global policy proposed by the WHO on the occasion of the II International Plan of Action on Aging approved by the United Nations General Assembly in 2002 that enables people to enhance their potential for physical, social, and mental well-being throughout the whole life course and to participate in society according to their needs, desires, and capacities and, at the same time, providing them with adequate protection, security, and care when required.

This new and positive vision of gerontology emerged supported by several scientific research data: 1) the broad plasticity of human organisms across life span, ${ }^{9,10}$ 2) the increase of variability of human characteristics across life span, ${ }^{11} 3$ ) the historical improvement of cognitive and physical characteristics across the last century, ${ }^{12,13}$ and finally, 4) the postponement of aging (from a decline and impairment perspective) at population level. ${ }^{14}$ This new paradigm is commonly expressed through the concept "active aging" with a wide global variation in the terms used to comprise the notion of "aging well", including active, successful, healthy, optimal, productive, positive, competent aging. ${ }^{15,16}$ Although all these terms can be considered synonymous, the concept of active aging and all the others lack a precise universally agreed definition and is commonly used to mean "all things to all people". ${ }^{17,18}$
This lack of scientific consensus has resulted in a variety of different applied strategies. Currently, there have been developed and implemented intervention programs devoted to promote active aging among older persons. Although it is a complex and heterogeneous concept, active aging is reduced to one outcome variable in most intervention programs: physical activity. In this sense, several interventions have been designed to promote active aging. For example, "Active Ageing Australia" promotes physical activity for a lifetime of health and well-being, considering that physical activity enriches persons' lives by supporting their ability to maintain independent, healthy lifestyles and participate in and contribute to the community. ${ }^{19}$ Other intervention programs encourage physical activity by offering a personal choicebased and telephone-assisted training program, ${ }^{20}$ enhancing pro-social behavior and volunteerism, ${ }^{21}$ or outdoor activities (such as biking), ${ }^{22}$ as strategies for promoting active aging.

On the other hand, some programs understand active aging only as emotional well-being, promoting life satisfaction. For example, through interventions centered in life review strategies on specific positive events ${ }^{23}$ or in education programs of care and exercises as strategies for enhancing emotional well-being, health, and therefore active aging. ${ }^{24}$

Under a different approach, intervention programs aim to promote active aging by enhancing social and intergenerational relationships ${ }^{25,26}$ or fostering empowerment and participation. ${ }^{27}$

Nevertheless, there are few documented intervention programs designed to promote active aging as a multidimensional concept as well as with empirical support.

Vital Aging ${ }^{\circledR}$ is an individual active aging promotion program that considers active aging as the lifelong adaptation process of maximizing four domains: 1) health and independence, 2) physical and cognitive functioning, 3) positive affect and control, and 4) positive engagement. ${ }^{28}$ This four-domain model of active aging has been tested through structural equation modeling with several samples and different methods ${ }^{29}$ and strongly supported by scientific literature through evaluation research. ${ }^{30-37}$ Moreover, developments from this program have had positive evaluation results such as the "I am Active program", involving a wider concept of active aging, including physical activity, healthy nutritional habits, cognitive functioning, and self-efficacy for aging, as core dimensions of active aging. ${ }^{38}$

Vital Aging (Vivir con Vitalidad) was initially developed in 1996 as a face-to-face course at the Autonomous University of Madrid. The objective of the program is to promote active aging through teaching basic knowledge about aging, promoting healthy behavioral lifestyles (physical exercise, nutrition), training strategies for optimizing 
cognitive functioning and compensating potential cognitive declines, optimizing positive affect, emotion, and control, and promoting social relationships and social engagement throughout the life course using new technologies.

Currently, the program has different versions:

1) Vital Aging face-to-face (VA-FF), participants attend 2/3-hour group sessions, held twice a week during 10 weeks. Sessions are conducted by an expert trainer.

2) Vital Aging-M (VA-M) is the multimedia version of the program where participants attend group sessions to watch video lessons on TV taught by European experts from Germany, Italy, and Spain (voices were translated to Spanish); a trained tutor is in charge of the DVD's management.

3) Vital Aging-eLearning (VA-eL) is the online version and was adapted cross-culturally; materials were designed to be used via Internet and implemented through the Learning Management System Moodle Platform. Students had a set of learning resources such as selfevaluation, reading, activities, forums, and tutorials. The course required $\sim 65$ hours during 3 months. It was implemented between 2010 and 2012.

4) Vital Aging-OCW (Open Course Ware), the course is supported by the Autonomous University of Madrid in the website http://ocw.uam.es/cursos/vivirconvitalidad/index. $\underline{\mathrm{html}}$; it is an open course available to any people around the world. For this version, eight video lessons were selected from the multimedia version materials, based on their relevance to promote active aging; each one includes practical and theoretical contents and a final assessment, as well as supporting materials. Course duration is 36 hours.

5) Vital Aging has also an Internet webpage http://www. envejecimientoactivo.es, where materials are available for older adults (only in Spanish), and in this way, they can be self-administrated or can be used by professionals and implemented as a structured program.

For more details about Vital Aging program versions, basic principles, and theoretical model. ${ }^{36}$

The first implemented version was VA-FF; it was developed at the Autonomous University of Madrid as an open life course and delivered in several editions since then. The program was later transformed into a multimedia version based on the administration of video lessons with the same structure and content as in the VA-FF.

Previous quasi-experimental studies have showed the effectiveness of all different versions. Outcome measures of active aging for all the studies included active life, perceptions of aging, physical activity, nutrition, health, social relationships, and life satisfaction. Supplementary material details information of previous outcomes of different editions of Vital Aging program, shown in Table S1. ${ }^{31-37}$

Finally, although active aging is a world concept defined by the WHO and tested cross-sectionally across the world, ${ }^{39}$ it continues being a cross-cultural challenge; thus, it is necessary to implement intervention strategies for helping older adults to reach this goal. In this sense, although the Vital Aging program is considered as an effective tool in European contexts, which has empirically demonstrated its efficacy to promote active aging through its different editions, versions, and target populations, ${ }^{36}$ the program has never been implemented in Latin American population. The objective of this study is to determine the effectiveness of the Vital Aging program to promote active aging in Mexican older adults.

\section{Methods}

\section{The intervention}

This is a quasi-experimental design with two experimental conditions; the sample was recruited by convenience (based on the institutional conditions and resources provided for the study). This is a semi-randomized study, ${ }^{40}$ participants were assigned to each experimental condition according to their choice, considering the schedule of the intervention. The study adopted an inter-group-intra-group comparison and pretest-posttest assessment.

As the first experimental condition, the VA-FF version was implemented, where a previously trained gerontologist conducted the sessions implemented as standard classes; supporting materials were taken from the basic texts "Vivir con Vitalidad" (Vital Aging). ${ }^{41}$ Nineteen 2-hour sessions were held twice a week during 10 weeks. The total duration of this version was 38 hours. Details of the lessons are shown in Table 1.

For the second experimental condition, the multimedia version was originally considered; however, important observations from previous experiences were taken into account. Due to technical issues when dubbing into Spanish, the videos originally in English or Italian were not applied directly as video lessons; instead of it, they were taught in the face-to-face format. For this reason, this second experimental condition was a combined version and named "Vital Aging combined" (VA-C) because it combines multimedia and face-to-face sessions of the program. However, it is important to underline that the program was mainly multimedia (11 lessons) and only one-third of it (seven lessons) was conducted in face-to-face format (Table 1).

VA-C procedures for implementation were followed according to each version. Lessons in multimedia format were implemented in a classroom equipped with TV, DVD, and a sound system; participants attended video lessons 
Table I Intervention lessons and format of the two versions of the Vital Aging ${ }^{\circledR}$ program applied in Mexico

\begin{tabular}{|c|c|c|c|}
\hline Domain & Lesson & VA-FF & VA-C \\
\hline \multirow[t]{5}{*}{ Behavioral health and independence } & Aging well & $\mathrm{F}$ & $M$ \\
\hline & Enjoy the control of your life & $\mathrm{F}$ & M \\
\hline & Regular exercise: the best formula for aging well & $\mathrm{F}$ & M \\
\hline & Taking care of your body: self-responsibility and self-management (i) & $\mathrm{F}$ & $\mathrm{F}$ \\
\hline & Nutrition and health: good food, good life (i) & $\mathrm{F}$ & $\mathrm{F}$ \\
\hline \multirow[t]{4}{*}{ Cognitive functioning } & Improve your memory & $\mathrm{F}$ & M \\
\hline & Train your mind: how to prevent brain aging (e) & $\mathrm{F}$ & $\mathrm{F}$ \\
\hline & The creative age (i) & $\mathrm{F}$ & $\mathrm{F}$ \\
\hline & Wisdom: the expression of lifelong learning (e) & $\mathrm{F}$ & $\mathrm{F}$ \\
\hline \multirow[t]{5}{*}{ Affect, control, and coping styles } & Self-efficacy perception & $\mathrm{F}$ & M \\
\hline & Positive thinking & $\mathrm{F}$ & M \\
\hline & Coping with stress & $\mathrm{F}$ & $M$ \\
\hline & Pleasant activities and well-being & $\mathrm{F}$ & M \\
\hline & Death is also part of life & $\mathrm{F}$ & M \\
\hline \multirow[t]{4}{*}{ Social participation and engagement } & How to improve human relationships (i) & $\mathrm{F}$ & $\mathrm{F}$ \\
\hline & Social support: the others need me too (i) & $\mathrm{F}$ & $\mathrm{F}$ \\
\hline & Sexuality: beyond genitality & $\mathrm{F}$ & M \\
\hline & Internet: a new system of communication & $\mathrm{F}$ & M \\
\hline
\end{tabular}

Notes: (i) Italian language; (e) English language.

Abbreviations: VA-FF, Vital Aging face-to-face; VA-C, Vital Aging combined; F, face-to-face format; M, multimedia format.

taught by experts from Spain. A trainer was in charge of the DVD's management; supporting materials were available for the participants in a Web site and they were encouraged to download them; lessons were followed by simultaneously watching $\mathrm{TV}$, listening to the expert, and reading the scripts. Lessons in face-to-face format were according to this version and described earlier. Multimedia sessions were carried out three times a week and face-to-face sessions twice a week. The total duration of the program was 30.5 hours ( 16.5 hours for multimedia and 14 hours for face-to-face sessions).

Contents and format of each session were implemented according to the four-domain model of active aging, and the detailed contents of each session were published elsewhere. ${ }^{36}$

In the control group, participants remained on a wait list, participating in the meantime in social activities organized by the senior center. After the study, they participated in the face-to-face version of the Vital Aging program if they were interested.

For both experimental conditions, VA-FF and VA-C, each session has a similar structure; it starts with a theoretical introduction of the lesson remarking its importance for a better aging based on scientific evidence. Later on, practical strategies are described and reviewed, and practical exercises are implemented. Finally, each session ends with some conclusion and feedback.

\section{Participants}

Participants were recruited from a senior center (Centro de Atención y Desarrollo Integral del Pensionado CADIP,
Dirección de Pensiones del Estado de Jalisco [Center for Comprehensive Care and Development of the Pensioner from the Direction of Pensions of the State of Jalisco]) for retired persons, former workers of the state government (eg, teachers). Participants lived in Guadalajara city, the capitol of Jalisco State located in the western region of Mexico.

Recruitment was carried out through the notice board and dissemination in social events in the center. Participants were included in the study only if they met the following criteria: 60 years and older, literate, and willing to participate. A written informed consent was obtained from all subjects. The Project was reviewed and approved by the Academic Board of the Doctoral Program on Behavioral Sciences, at Psychology Faculty, University Autonomous of Madrid.

The total sample was composed of 90 older persons, who were assigned to an experimental condition (Figure 1). The sample size was determined by convenience, limited by the capacity, schedule, and resources provided by the senior center. Due to institutional conditions, intervention was carried out in two trials; in a first trial, VA-FF, VA-C, and a control group were included, and in a second trial, VA-FF and a control group were included; $n=18$ participants composed each group. For this study, participants in the two VA-FF groups, as well as the two control groups, were joined for analyses purposes, and there was no difference between the groups. The total groups included VA-FF $=36, \mathrm{VA}-\mathrm{M}=18$, and control group $=36$ participants.

The final sample was composed of $n=76$, all women with an age range of 60-84 years (mean age $=65.66$ years, 


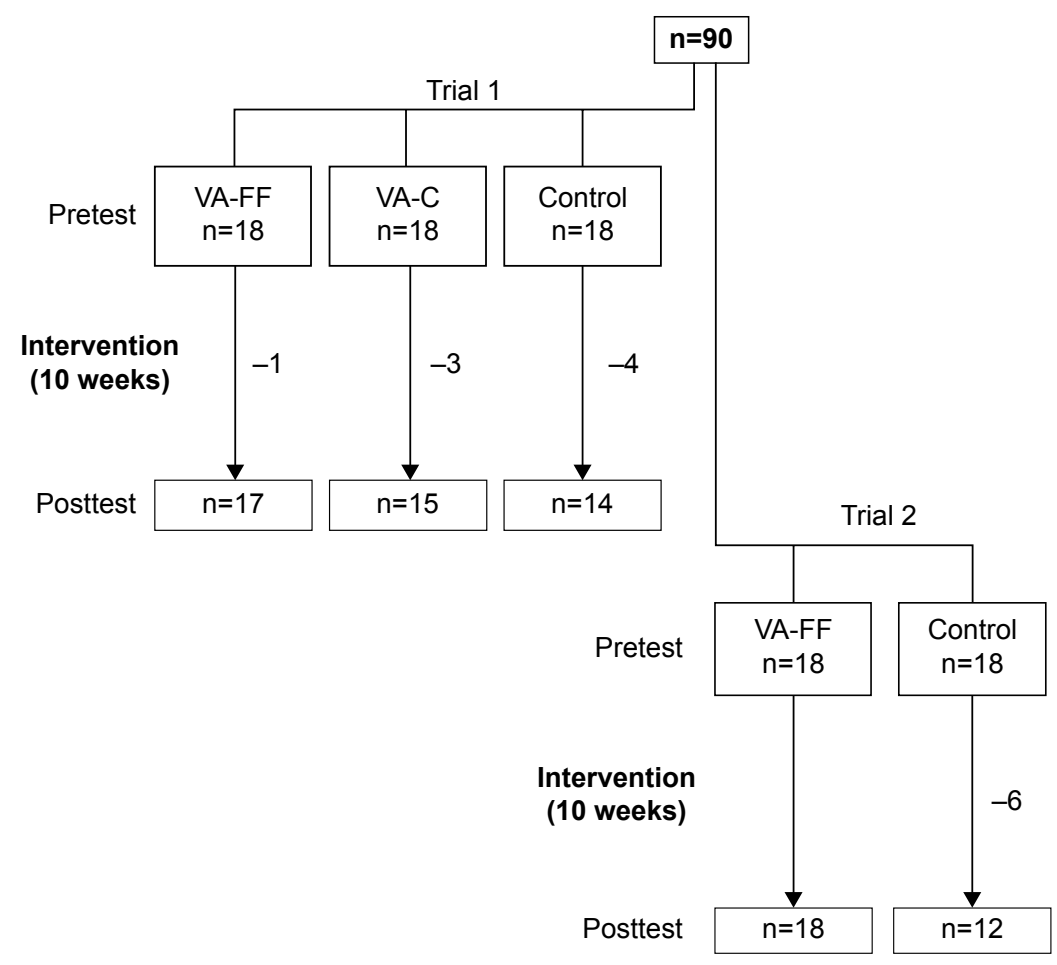

Figure I Flow chart of the participants in the study.

Abbreviations: VA-FF, Vital Aging face-to-face; VA-C, Vital Aging combined version.

$\mathrm{SD}=6.48$ years $), \mathrm{VA}-\mathrm{FF}$ version involved $\mathrm{n}=35$ participants (mean age $=64.7$ years, $\mathrm{SD}=5.8$ years), VA-C version $\mathrm{n}=15$ (mean age $=63.9$ years, $\mathrm{SD}=4.5$ years), and control group $\mathrm{n}=26$ (mean age $=67.8$ years, $\mathrm{SD}=6.4$ years). Attrition for each experimental condition was $\mathrm{VA}-\mathrm{FF}=2.77 \%$, VA-C $=16.7 \%$, and control $=27.8 \%$. There were no differences in age and education between participants and those who did not complete the study; their exclusion from both the study and the analysis did not alter the similarity between groups.

\section{Procedure}

A pretest was conducted for outcome measures, then intervention was developed during 10 weeks, and the posttest was conducted at the end of the program. A formative evaluation was also included, at the end of each lesson, for exploring participants' opinions about materials, difficulty, format, and general appreciation of the lesson and their satisfaction.

\section{Outcome measures}

Participants from VA-FF, VA-C, and control groups were evaluated at baseline and posttest. A previously trained psychologist applied an assessment battery. Personal appointments were made, and participants were interviewed at the senior center. For comparison purposes, the assessment was based on a battery designed for the program and used in previous studies; these variables were considered primary outcomes, which include the following:

1) Active life explores the frequency of performing 24 different activities: intellectual (eg, reading, solving crossword puzzles or puzzles), art (eg, studying singing, playing an instrument), social (eg, caring someone else, visiting friends), and domestic (eg, cleaning the house, watching TV). Response options range from nothing $=1$, some $=2$, much $=3$, and pretty much $=4 .{ }^{42}$

2) Perceptions of aging explores the degree of agreement with 16 positive statements of aging; typically, they include topics related to aging such as autonomy (eg, I will solve problems when they appear), memory (eg, my memory is as good as before), attitude (eg, life is always worth), and perception of one's action (eg, I am able to learn something new). Response options range from $1=$ strongly disagree to $4=$ strongly agree, the higher the score the better the opinion.

3) Physical exercise explores the level and frequency of physical activity done in the last month; answers range from $1=$ sedentary to $5=$ intense exercise $>3$ times a week. ${ }^{43}$

4) Frequency of social relationships; this scale explores the frequency of social activities with family, friends, and neighbors. Response options range from $1=$ less than once a month to $5=$ several times a week. ${ }^{44}$ 
5) Satisfaction with social relationships is a measure about the degree of satisfaction about social relationships with family, friends, and neighbors $(1=$ not satisfied to $5=$ very satisfied). ${ }^{44}$

6) Life satisfaction refers to the current level of satisfaction about the own life expressed by the person after considering both the good and the bad things he or she had lived. The scale ranges from $1=$ none to $4=$ very much, where a higher score indicates greater life satisfaction.

7) Self-efficacy for aging assesses the people's beliefs about their own capability to solve or cope future problems related to the own health, family, abilities, and functionality. It includes 10 items such as "I will maintain my intellectual functioning such as now" and "I could solve a health problem if emerged". Answer options range from $1=$ strongly disagree to $4=$ strongly agree, where a higher score means better self-efficacy for aging. ${ }^{43}$

In addition, the following secondary outcomes were assessed for all participants in order to explore a broader impact of the program:

1) Subjective health explores the current self-perception of health, considered as $4=$ very good, $3=$ good, $2=$ regular, $1=$ poor.

2) Memory objective performance, measured by Digit Span Backward Subtest. ${ }^{45}$

3) Frequency of memory problems explores how often people report forgetfulness on a variety of daily activities, such as losing the house keys, doing activities, recognizing people, etc. The range of answers implies that this happens $1=$ never to $5=$ very often.

\section{Data analyses}

Statistical analyses were performed with SPSS software version 18 (Chicago, IL, USA). Data were processed to obtain descriptive statistics (proportions, mean value, and standard deviation) from demographic and outcome variables. Intergroup comparisons were tested by one-way analysis of variance (ANOVA). A repeated-measures ANOVA with a Greenhouse-Geisser correction was conducted to determine if means in outcome variables differed statistically significantly between time points (pretest and posttest). Cohen's $d$ was estimated to determine the effect size classified as small $(d=0.2)$, medium $(d=0.5)$, and large $(d \geq 0.8) .{ }^{46}$ For formative evaluation, data from 19 sessions were averaged for each version of the program.

\section{Results}

The socio-demographic characteristics of participants in the two experimental and the control groups are shown in Table 2. The groups were comparable for age, marital status, and education.

The evaluation study starts by a formative evaluation. With this purpose, both versions of the program were compared in order to know older adults' perception about different conditions of each version (lesson characteristics, proposed exercises, trainer abilities, graphics and images presented, understanding and doubts, duration, general satisfaction, and usefulness) of the Vital Aging program as well as their satisfaction. Results are shown in Table 3. A few but important differences between the two experimental conditions can be observed. Participants in the combined version (VA-C) considered that the lessons reviewed in each session during the program were more interesting and new, compared to those who participated in the face-to-face (VA-FF) version $(P<0.05)$. In the same sense, they evaluated both the exercises and the examples used during the program as more interesting $(P<0.05)$. In general, older adults who participated in the version VA-C (which combined multimedia and

Table 2 Socio-demographic characteristics of the participants

\begin{tabular}{|c|c|c|c|c|}
\hline Variable & $\begin{array}{l}\text { VA-FF } \\
(n=35), \%(n)\end{array}$ & $\begin{array}{l}\text { VA-C } \\
(n=15), \%(n)\end{array}$ & $\begin{array}{l}\text { Control } \\
(n=26), \%(n)\end{array}$ & $P$-value \\
\hline Age, years (mean $\pm S D)$ & $64.7 \pm 5.8$ & $63.9 \pm 4.5$ & $67.8 \pm 6.4$ & $0.156^{\mathrm{a}}$ \\
\hline $60-64$ & $62.9(22)$ & $60.0(9)$ & $46.2(12)$ & $0.368^{\mathrm{b}}$ \\
\hline $65-69$ & $20.0(7)$ & $26.7(4)$ & $26.9(7)$ & \\
\hline $70-74$ & $5.7(2)$ & $13.3(2)$ & $3.8(I)$ & \\
\hline $75+$ & $11.4(4)$ & $0.0(0)$ & $23.1(6)$ & \\
\hline Marital status & & & & $0.447^{\mathrm{b}}$ \\
\hline Married & $33.4(12)$ & $53.3(8)$ & $38.5(10)$ & \\
\hline Single ${ }^{c}$ & $65.7(23)$ & $46.7(7)$ & $61.5(16)$ & \\
\hline Education & & & & $0.204^{b}$ \\
\hline High school and below & $23.5(9)$ & $6.7(1)$ & $30.8(8)$ & \\
\hline Above high school & $76.5(26)$ & $93.4(14)$ & $69.2(18)$ & \\
\hline
\end{tabular}

Notes: aANOVA. 'Pearson's chi-square. 'Includes never married, widow, divorced, separated.

Abbreviations: SD, standard deviation; ANOVA, analysis of variance; VA-FF, Vital Aging face-to-face; VA-C, Vital Aging combined. 
Table 3 Comparison of the formative evaluation in face-to-face and combined versions

\begin{tabular}{|c|c|c|c|}
\hline Variable & Face-to-face & $\begin{array}{l}\text { Combined } \\
\text { (face-to-face/ } \\
\text { multimedia) }\end{array}$ & Total \\
\hline \multicolumn{4}{|l|}{ Lesson ( $\mathrm{I}=$ strongly disagree to $4=$ strongly agree) } \\
\hline Interest & 3.80 & $3.89 *$ & 3.83 \\
\hline Difficulty & 1.35 & 1.50 & 1.39 \\
\hline Usefulness for daily living & 3.73 & 3.77 & 3.74 \\
\hline Novelty & 3.26 & $3.43 *$ & 3.31 \\
\hline \multicolumn{4}{|l|}{ Exercises and examples ( $I=$ strongly disagree to $4=$ strongly agree $)$} \\
\hline Interesting & 3.73 & $3.83^{*}$ & 3.76 \\
\hline Useful & 3.72 & 3.77 & 3.74 \\
\hline Complicated & 1.45 & 1.49 & 1.46 \\
\hline \multicolumn{4}{|l|}{ Trainer $(I=$ strongly disagree to $4=$ strongly agree $)$} \\
\hline Mastery on the lesson & 3.78 & 3.70 & 3.76 \\
\hline Clear description & 3.79 & 3.69 & 3.76 \\
\hline Ability to attract attention & 3.78 & 3.76 & 3.77 \\
\hline \multicolumn{4}{|l|}{ Graphics ( $I=$ strongly disagree to $4=$ strongly agree $)$} \\
\hline Useful/facilitate understanding & 3.71 & 3.71 & 3.71 \\
\hline \multicolumn{4}{|l|}{ Images ( $I=$ strongly disagree to $4=$ strongly agree $)$} \\
\hline Useful/facilitate understanding & 3.70 & 3.72 & 3.71 \\
\hline \multicolumn{4}{|l|}{ Understanding ( $\mathrm{I}=$ strongly disagree to $4=$ strongly agree $)$} \\
\hline I think I understood the lesson & 3.63 & 3.61 & 3.62 \\
\hline \multicolumn{4}{|l|}{ Doubts ( $I=$ strongly disagree to $4=$ strongly agree $)$} \\
\hline Do you have questions to the trainer? & 1.26 & 1.22 & 1.25 \\
\hline Duration (3 excessive to I appropriate) & 1.99 & 1.98 & 1.98 \\
\hline Global evaluation ( $\mathrm{I}$ minimum to 10 maximum) & 9.85 & 9.85 & 9.85 \\
\hline General satisfaction ( $\mathrm{I}=$ strongly disagree to $4=$ strongly agree $)$ & 3.64 & $3.77^{*}$ & 3.68 \\
\hline $\begin{array}{l}\text { They consider that the knowledge gained in this program has been or will } \\
\text { be useful in their daily lives ( } I=\text { strongly disagree to } 4=\text { strongly agree })\end{array}$ & 2.50 & $2.88^{*}$ & 2.72 \\
\hline
\end{tabular}

Notes: $* P<0.05$ (Student's t-test). Data are shown as mean.

face-to-face sessions) reported being more satisfied with the program, compared to those participating only in the faceto-face sessions $(P<0.05)$.

Table 4 shows the effect size of outcome variables. The results of inter-group comparisons showed that there were no significant differences in pretest in almost all variables. Differences between VA-FF and control groups were found only in active life $(P<0.01)$ and perception of aging $(P<0.001)$, where the control group had a better score. However, these differences were not maintained in the posttest comparison due to the significant improvement of VA-FF after the intervention. Inter-group comparisons in the posttest showed no significant differences between groups in any variable; only in the memory performance, VA-FF group reported better performance than the control group $(P<0.05)$.

On the results of intra-group comparisons, regarding the performance of activities or active life, participants in the VA-C version reported a significant increase in frequency of activities $\left(F_{1,11}=7.427, P=0.02\right.$, partial $\left.\eta^{2}=0.403\right)$, with a medium effect size of $d=0.68$; in the VA-FF group, a trend of improvement in activities was observed $\left(F_{1,30}=3.805, P=0.06\right.$, partial $\left.\eta^{2}=0.113\right)$ with a small effect size $(d=0.25)$, while the control group remained with no significant changes.

Perceptions on aging improved significantly in participants of both VA-FF $\left(F_{1,30}=121.41, P=0.000\right.$, partial $\left.\eta^{2}=0.802\right)$ and VA-C $\left(F_{1,11}=55.298, P=0.000\right.$, partial $\left.\eta^{2}=0.834\right)$ versions, with very large effect sizes of $d=1.95$ and $d=2.10$, respectively. No significant changes were reported in the control group $(P=0.255)$.

No effects in physical activity, subjective health, or life satisfaction were found in either versions of the Vital Aging program $(P>0.05)$, although a medium effect size in life satisfaction was observed $(d=0.51)$ in the VA-C group.

Regarding social relationships, although a significant increase on the frequency of relationships with family, friends, and neighbors was not observed $(P>0.05)$, participants of the living version VA-FF reported higher satisfaction in their social relationships after the program $\left(F_{1,30}=7.142\right.$, $P=0.013$, partial $\eta^{2}=0.215$ ), with a medium effect size of $d=0.49$, meanwhile participants in the combined version and the control group remained without significant changes. 
Table 4 Effect sizes of outcome measures of the two versions of Vital Aging program (VA-FF, n=35; VA-C, n=I5; control, n=26)

\begin{tabular}{|c|c|c|c|c|c|}
\hline Variable & Group & Pretest & Posttest & $P$-value & $d$ \\
\hline \multirow[t]{3}{*}{ Active life } & VA-FF & $2.10 \pm 0.4 I^{* *}$ & $2.20 \pm 0.38$ & 0.060 & 0.25 \\
\hline & VA-C & $2.21 \pm 0.35$ & $2.50 \pm 0.50$ & 0.020 & 0.68 \\
\hline & Control & $2.40 \pm 0.55 * *$ & $2.24 \pm 0.53$ & 0.083 & 0.30 \\
\hline \multirow[t]{3}{*}{ Perceptions of aging } & VA-FF & $2.63 \pm 0.39 * * *$ & $3.37 \pm 0.37$ & 0.000 & 1.95 \\
\hline & VA-C & $2.82 \pm 0.25$ & $3.47 \pm 0.37$ & 0.000 & 2.10 \\
\hline & Control & $2.91 \pm 0.26 * * *$ & $3.07 \pm 0.53$ & 0.255 & 0.41 \\
\hline \multirow[t]{3}{*}{ Physical activity } & VA-FF & $2.68 \pm 1.40$ & $2.68 \pm 1.35$ & 1.000 & 0.00 \\
\hline & VA-C & $3.08 \pm 1.08$ & $3.08 \pm 0.90$ & 1.000 & 0.00 \\
\hline & Control & $2.69 \pm 1.53$ & $3.06 \pm 1.48$ & 0.232 & 0.25 \\
\hline \multirow[t]{3}{*}{ Life satisfaction } & VA-FF & $3.20 \pm 0.61$ & $3.30 \pm 0.65$ & 0.375 & 0.16 \\
\hline & VA-C & $3 .|7 \pm 0.7|$ & $3.58 \pm 0.90$ & 0.096 & 0.51 \\
\hline & Control & $3.38 \pm 0.7 \mid$ & $3.56 \pm 0.72$ & 0.333 & 0.25 \\
\hline \multirow[t]{3}{*}{ Frequency of social relationships } & VA-FF & $3.85 \pm 1.14$ & $4.07 \pm 1.10$ & 0.386 & 0.20 \\
\hline & VA-C & $3.33 \pm 1.43$ & $3.75 \pm 1.21$ & 0.241 & 0.32 \\
\hline & Control & $3.75 \pm 1.43$ & $3.62 \pm 1.36$ & 0.609 & 0.09 \\
\hline \multirow[t]{3}{*}{ Satisfaction with social relationships } & VA-FF & $3.32 \pm 0.78$ & $3.7 I \pm 0.82$ & 0.013 & 0.49 \\
\hline & VA-C & $3.30 \pm 0.7 \mid$ & $3.66 \pm 0.66$ & 0.097 & 0.53 \\
\hline & Control & $3.52 \pm 1.01$ & $3.60 \pm 0.91$ & 0.742 & 0.08 \\
\hline \multirow[t]{3}{*}{ Subjective health } & VA-FF & $3.03 \pm 0.73$ & $3.17 \pm 0.65$ & 0.380 & 0.20 \\
\hline & VA-C & $3.00 \pm 0.77$ & $3.09 \pm 0.83$ & 0.769 & 0.11 \\
\hline & Control & $2.69 \pm 1.01$ & $2.75 \pm 1.12$ & 0.817 & 0.06 \\
\hline \multirow[t]{3}{*}{ Memory performance } & VA-FF & $5.28 \pm 1.16$ & $5.79 \pm 0.90 *$ & 0.019 & 0.50 \\
\hline & VA-C & $5.09 \pm 1.13$ & $5.09 \pm 1.30$ & 1.000 & 0.00 \\
\hline & Control & $5.06 \pm 1.12$ & $4.8 \mathrm{I} \pm 1.83^{*}$ & 0.483 & 0.17 \\
\hline \multirow[t]{3}{*}{ Frequency of memory problems } & VA-FF & $2.7 I \pm 0.63$ & $2.54 \pm 0.58$ & 0.074 & 0.28 \\
\hline & VA-C & $2.92 \pm 0.47$ & $2.79 \pm 0.70$ & 0.551 & 0.22 \\
\hline & Control & $2.63 \pm 0.86$ & $2.43 \pm 1.05$ & 0.345 & 0.21 \\
\hline \multirow[t]{3}{*}{ Self-efficacy for aging } & VA-FF & $2.72 \pm 0.53$ & $3.09 \pm 0.46$ & 0.000 & 0.75 \\
\hline & VA-C & $2.98 \pm 0.67$ & $3.36 \pm 0.6 \mathrm{I}$ & 0.000 & 0.59 \\
\hline & Control & $2.90 \pm 0.32$ & $3.02 \pm 0.77$ & 0.333 & 0.22 \\
\hline
\end{tabular}

Notes: $d$ is the effect size; data are shown as mean \pm standard deviation. For inter-group comparisons: $* P<0.05, * * P<0.01$, $* * * P<0.001$. Abbreviations: VA-FF, Vital Aging face-to-face; VA-C, Vital Aging combined.

Considering the effects of the program on the participant's memory, those in the VA-FF version reported a significant improvement on their memory performance after the intervention $\left(F_{1,30}=67.77, P=0.019\right.$, partial $\left.\eta^{2}=0.708\right)$, with a medium effect size of $d=0.50$, and a trend to report less frequency of memory problems $\left(F_{1,30}=3.429, P=0.074\right.$, partial $\eta^{2}=0.106$ ), with a small effect size of $d=0.28$. In contrast, those who participated in the VA-C version and the control group remained without significant changes in memory outcomes.

On the other hand, both VA-FF $\left(F_{1,30}=29.265, P=0.000\right.$, partial $\left.\eta^{2}=0.520\right)$ and VA-C $\left(F_{1,11}=34.385, P=0.000\right.$, partial $\eta^{2}=0.775$ ) reported a significant improvement in selfefficacy for aging, with the effect sizes being large for both versions of the program ( $d=0.75$ and $d=0.59$, respectively). No change was observed in the control group.

\section{Conclusion}

The study's findings show that the Vital Aging program in the "face-to-face" and "combined" (multimedia/ face-to-face) versions improves some active aging indicators or outcomes in Mexican older persons. Specifically, after both versions, participants reported significant higher frequency of cultural, intellectual, and social activities than the control group and had better views about aging, more satisfaction with social relationships, and better self-efficacy for aging. Older adults in VA-FF also reported better memory performance, a better perception of it, and a trend to have less memory problems, while older persons in VA-C showed a trend to have better life satisfaction.

These results are, in general, similar to those in Spanish editions, which reveal the consistency of the program in different populations and place the program as an effective cross-cultural intervention to promote active aging.

Specifically, in this study, it was found as an outcome the increase of activities or active life in older adults participating in the program; this finding is consistent with the reports of previous editions, whether in a face-to-face ${ }^{33,34}$ or multimedia version. ${ }^{30-32,37}$ In addition to this evidence, a version combining both formats is also effective. The fact 
of being involved on a variety of activities (cultural/artistic, intellectual, social) is important in old age, due to its impact on different dimensions of life. For example, in a review that analyzed qualitative, quantitative, and mixed methods, including different artistic activities such as music and painting, it was found that artistic engagement could contribute to health and quality of life in older adults. ${ }^{47}$ In the same way, it has been reported that active participation in arts such as dance, expressive writing, music (singing and instrumental), theater arts, and visual arts has been related to mental and physical improvements in memory, creativity, problem solving, everyday competence, reaction time, balance/gait, and quality of life. ${ }^{48}$

A remarkable finding in this study is the improvement in perceptions of aging, which is consistent in all previous editions of the Vital Aging program, whether face-to-face or multimedia versions, in community or nursing home settings. ${ }^{30-35}$ Besides, in this study, it was found that a combined (VA-C) version is also effective in promoting positive views about aging. This is a significant outcome of the program because the evidence from other studies has shown that the ideas older adults hold about themselves, as aging people, have an influence on their own aging process. Selfperception of aging is strongly influenced by the beliefs held both by the society about older adults and by themselves about the group of older adults. ${ }^{28}$ In this sense, research has shown that ideas about aging were good predictors of health and self-perception of aging predicts longevity. ${ }^{49}$ Moreover, it has been found that individuals with positive self-perception of aging lived an average of 7.5 years longer than those with negative self-perceptions of aging. ${ }^{50}$ In this sense, results of this study had shown that Vital Aging program encourages positive beliefs about aging (in general) and the own aging process (in particular), which finally promotes a healthy and active aging. In sum, there is strong evidence that the improved perception of aging is associated with health and longevity.

Regarding the impact of the Vital Aging program in social relationships, this study failed (as well as previous editions) in promoting more frequent social relationships, perhaps because the Vital Aging program participants had previously strong social ties. Nevertheless, in this study, it was found that the group who participated in the face-to-face version reported greater satisfaction with social relationships. This result may be influenced due to the positive and good relationship among the participants in the group, but this is difficult to distinguish by the program effect itself. Particularly, the Unit "Social participation and engagement" proposes very specific guidelines on how to improve social relationships (including assertive communication with practical exercises, for example), underlines the importance of providing social support to others (not only to receive it), and emphasizes the need of the active participation of older adults in the society and its impact for the other generations. The lessons of this Unit were taught in face-to-face format for both intervention groups and seem to have had an effect on a greater social relationships satisfaction.

The effects of the Vital Aging program related to the physical activity are controversial when compared to previous editions. In this study, no significant effects were found, while in previous studies, significant results were reported only in the multimedia format and when it is applied specifically in community settings. ${ }^{30,32}$ Physical activity is a complex behavior to promote, which involves a complex and elaborate process of decision making. ${ }^{51}$ Commonly, health promotion interventions seek to increase levels of physical activity by influencing personal and educational factors that contribute to physical activity behavior. However, the determinants and background of physical activity are diverse and include awareness of the physical activity message and its benefits, attitudes, and intentions toward being active, as well as supra-individual factors such as policies, environments, and cultural norms that facilitate physical activity. ${ }^{52}$ These factors should be considered in order to have a better impact in promoting physical activity, due to its importance to reach active aging.

Additionally, in this study, outcome variables that have not been studied previously were included, in order to explore the effects of the program in other important domains of active aging. Variables were memory (objective and subjective) and self-efficacy for aging. Both reported improvements only in the face-to-face format of the Vital Aging program.

Self-efficacy is defined as people's beliefs about their capabilities to produce designated levels of performance that exercise influence over events that affect their lives. Perceived self-efficacy beliefs determine how people feel, think, motivate themselves, and behave. Such beliefs produce these diverse effects through four major processes. These include cognitive, motivational, affective, and selection processes. ${ }^{53}$

Specifically, self-efficacy for aging has been studied in the Vital Aging program to examine to what extent it can be trained and improved. In this context, self-efficacy for aging involves the conviction for achieving a well aging by preventing and solving health problems, maintaining physical function (independence), controlling emotions, solving social relationships problems (if they appear), memory and cognitive issues, and the sense of being capable of accomplishing a well aging in the future. In sum, self-efficacy 
beliefs are strongly related to successful aging because they contribute to perceive age-related situations not as threats but as challenges, and they also support individuals to remain committed to selected goals, and self-efficacy perceptions seem to have strong associations with health and survival. ${ }^{28}$ In this study, positive outcomes with the largest effect sizes suggest that older persons strongly believe that they can achieve an active aging, and this is an important predictor of their ability to do so; due to the sense of self-efficacy, it can even be considered precedent of behavior. ${ }^{54}$ Therefore, the perception of self-efficacy for aging is related to the process of active aging. ${ }^{28}$

Regarding the memory improvements, results of the Vital Aging program are relevant due to memory, and specifically working memory, that is considered a basic mechanism that explains age-related decline in cognitive function, ${ }^{55}$ and subjective memory is considered a predictor of the performance of memory itself. ${ }^{56}$ Moreover, differences of the effectiveness between versions could be explained by the format itself. Although face-to-face version implies a major interaction between trainers and classmates, a combined format (multimedia and face-to-face) introduces variability in techniques and strategies for education and training, which could be more stimulating and innovative for older adults, besides the idea of being involved in an international program with international trainers (evident in lesson taught by Spaniard or Italian experts). This fact could explain that the version combining multimedia and living format was better evaluated, and participants reported more satisfaction than those participating only in face-to-face sessions.

The limitations of this study include that the generalization of the results may be limited by the inclusion criteria and the characteristics of the participants; in this case, they could be considered with exceptional conditions and do not necessarily represent the Mexican older adults. Participants were only women, had higher education, and received a pension; these conditions could represent an advantage and facilitate better conditions to get benefits from the program. However, the original version of Vital Aging was designed for older adults with these characteristics (Spaniards), and then this represents a first approach, which allows a realistic idea about how the program should be adapted for being successfully implemented in a different population and being a cross-cultural effective program. Sample size might also be a limitation; in this sense, further interventions are required involving larger samples. Due to this study not incorporating any blinding procedures, it must be acknowledged that the likelihood of differential treatment or assessment of outcomes, may represent bias. However, other methodologic precautions recommended to minimize potential bias have been incorporated, such as ensuring that groups involved were treated equally, and outcomes were measured as objectively as possible..$^{57}$ These limitations may influence that the program still have a modest impact on some outcome variables; however, this is a first approach to measuring the effectiveness of the Vital Aging program as a cross-cultural applied strategy to promote active aging, where the strengthens and challenges of the intervention begin to being identified.

Finally, active aging might be considered as a global strategy in the sense that it could be good for citizens of all ages because it promotes healthy life styles, but also, in terms of maximizing personal development and empowerment. Therefore, it could increase the quality of life by getting the best from human capital, extending community participation and solidarity, avoiding intergenerational conflicts, and creating a fairer, more inclusive society. Active older persons are a resource for their families, their communities, and the economy, ${ }^{5}$ although there is almost no acknowledgment of the substantial positive contributions and potential productivity of an aging society. ${ }^{2}$

Aging as a human process is not a random phenomenon, the individual is an agent of his/her own aging process, in this sense, the capacity for aging well, healthy, and active comes, in a certain extent, from decisions taken by individuals themselves as well as their behavioral repertoires learnt across the life span. ${ }^{6}$ According to this statement, consistent results of the Vital Aging program (both face-to-face or combined format) place it as a useful cross-cultural tool for promoting active aging in older adults.

\section{Acknowledgment}

Project PSI2014-52464-P from the Ministry of Economy and Competitiveness (MINECO) in Spain supported this work.

\section{Disclosure}

The authors report no conflicts of interest in this work.

\section{References}

1. United Nations. World Population Situation in 2014. New York, NY: United Nations; 2014.

2. Rowe JW. Successful aging of societies. J Am Acad Arts Sci. 2015; 144(2):5-12.

3. Prohaska TR, Anderson LA, Binstock RH. Public Health for an Aging Society. Baltimore, MA: Johns Hopkins University Press; 2012.

4. World Health Organization. World Report on Ageing and Health. Luxemburg: WHO; 2015.

5. Walker A. A strategy of active ageing. Int Soc Secur Rev. 2002;55: 121-139. 
6. Fernández Ballesteros R, Robine MA, Walker A, Kalache A. Active aging: a global goal. Curr Gerontol Geriatr Res. 2013;2013: 298012.

7. World Health Population. Active Ageing. Geneva, Switzerland: World Health Organization; 2002.

8. International Longevity Centre ILC. Active Ageing: A Policy Framework in Response to the Longevity Revolution. Brazil: ILC; 2015.

9. Lerner RM. On the Nature of Human Plasticity. New York, NY: Cambridge University Press; 1984.

10. Fernández-Ballesteros R, Botella J, Zamarrón MD, et al. Cognitive plasticity in normal and pathological aging. Clin Interv Aging. 2012;7: $15-25$.

11. Stawski RS, Smith J, MacDonald SWS. Intraindividual variability and covariation across domains in adulthood and aging: contributions to understand behavior, health and development. In: Dielh M, editor. Handbook of Intraindividual Variability Across the Life Span. New York, NY: Routledge; 2015:xix, 371.

12. Schaie KW. Developmental Influences on Adult Intelligence: The Seattle Longitudinal Study. New York, NY: Oxford University Press 2005.

13. Schaie KW. What can we learn from longitudinal studies of adult development? Res Hum Dev. 2005;2:133-158.

14. Vaupel JW, Carey JP, Christensen K, et al. Biodemographic trajectories of longevity. Science. 2009;280:855-860.

15. Fernández-Ballesteros R. Geropsychology. European Perspectives for an Ageing World. Gottingën: Hogrefe \& Huber; 2007.

16. Fernández-Ballesteros R, Molina MA, Schettini R, Santacreu M. The semantic network of aging well. In: Robine J-M, Jagger C, Crimmins EM, editors. Healthy Longevity. Annual Review of Gerontology and Geriatrics. Vol. 33. New York, NY: Springer; 2013.

17. Foster L, Walker A. Active and successful aging: a European policy perspective. Gerontologist. 2015;55(1):83-90.

18. Walker A, Maltby T. Active ageing: a strategic policy solution to demographic ageing in the European Union. Int J Soc Welf. 2012;17: 117-130.

19. Active Ageing Australia [home page on the Internet]. Adelaide, Australia: Government of South Australia [updated December 2015]. Available from: http://www.activeageingaustralia.com.au. Accessed May 30, 2016.

20. Hooker SP, Seavey W, Weidmer CE, et al. The California active aging community grant program: translating science into practice to promote physical activity in older adults. Ann Behav Med. 2005; 29(3):155-165.

21. Foy CG, Vitolins MZ, Case L, et al. Incorporating prosocial behavior to promote physical activity in older adults: rationale design of the Program for Active Aging and Community Engagement (PACE). Contemp Clin Trials. 2013;36(1):284-297.

22. Boyes M. Outdoor adventure and successful ageing. Ageing Soc. 2013; 33:644-665.

23. Latorre JM, Serrano JP, Ricarte J, Bonete B, Ros B, Sitges E. Life review based on remembering specific positive events in active aging. J Aging Health. 2015;27(1):140-157.

24. Estebsari F, Taghdisi MH, Foroushani AR, Ardebili EA, Shojaeizadeh D. An educational program based on the successful aging approach on health-promotion behaviors in the elderly: a clinical trial study. Iran Red Crescent Med J. 2014;16(4):16314.

25. Horowitz BP, Wong SD, Dechello K. Intergenerational service learning: to promote active aging, and occupational therapy gerontology practice. Gerontol Geriatr Educ. 2010;31(1):75-91

26. Sikora SA. The University of Arizona College of Medicine optimal aging program: stepping in the shadows of successful aging. Gerontol Geriatr Educ. 2006;27(2):59-67.

27. Martinez-Maldonado ML, Correa Muñoz E, Mendoza-Nuñez VM. Program of active aging in a rural Mexican community: a qualitative approach. BMC Public Health. 2007;7:276.

28. Fernández Ballesteros R. Active Aging: The Contribution of Psychology. Göttingen: Hogrefe; 2008.
29. Fernández Ballesteros R, Schettini R, Molina MA, Santacreu M. Testing a Four Domain Model of Aging Well. Madrid: Autonomous University of Madrid; 2012.

30. Fernández-Ballesteros R, Caprara MG, García LF. Vivir con Vitalidad-M: un programa europeo multimedia [Vital aging-M: an European multimedia program]. Int Soc. 2004;13:63-85. Spanish.

31. Fernández-Ballesteros R, Caprara MG, Iñiguez J, García LF. Vital aging-M: an European multimedia programme. Psychol Spain. 2005; 9:1-12.

32. Fernández-Ballesteros R. Evaluation of "Vital Aging-M": a psychological program for promoting optimal aging. Eur Psychol. 2005;10(2): $146-156$.

33. Caprara MG. Envejecimiento con éxito: valoración de un programa [Successful aging: evaluation of a program] [PhD thesis]. Madrid: Autonomus University of Madrid; 2005.

34. Fernández-Ballesteros R, Caprara MG, Iñiguez J, García LF. Promoción del envejecimiento activo: efectos del programa «Vivir con vitalidad $\rangle^{{ }^{R}}$ [Promoting active ageing: effects of the «Vital living» programme]. Rev Esp Geriatr Gerontol. 2005;40(2):92-102.

35. Caprara MG, Fernandez-Ballesteros R. Promoting Active Aging: New Effects of Vital Aging Program. Madrid: UDIMA; 2012.

36. Caprara MG, Molina MA, Schettini R, et al. Active aging promotion: results from the Vital Aging Program. Curr Gerontol Geriatr Res. 2013; 2013:817813.

37. Caprara MG, Fernández-Ballesteros RF, Alessandri G. Promoting aging well: evaluation of Vital-Aging-Multimedia Program in Madrid, Spain. Health Promot Int. 2016;31(3):515-522.

38. Mendoza-Ruvalcaba NM, Arias-Merino ED. "I am active": effects of a program to promote active aging. Clin Interv Aging. 2015;10: 829-837.

39. Fernández-Ballesteros R, García LF, Abarca D, et al. Lay concept of aging well: cross-cultural comparisons. J Am Geriatr Soc. 2008;56(5): 950-952.

40. Silverman WA. Patient preferences and randomized trials. Lancet. 1994;344(8928):1023.

41. Fernández-Ballesteros. Vivir con Vitalidad [Vital Aging]. Madrid: Pirámide; 2002.

42. Díez-Nicolás J. Los Mayores en la Comunidad de Madrid [The elderly in the community of Madrid]. Spain: Obra Social Caja de Madrid; 1996.

43. Fernández-Ballesteros R, Zamarrón MD, Rudinger G, et al. Assessing competence: the European survey on aging protocol (ESAP). Gerontology. 2004;50(5):330-347.

44. Diaz-Veiga P. Assessment of social support [Evaluación del apoyo social]. In: Fernandez-Ballesteros R, editor. El Ambiente: Análisis Psicológico [The enviroment: psychological analyses]. Madrid: Pirámide; 1987:125-149.

45. Wechsler D. Wechsler Adult Intelligence Scale. Cuauhtémoc, México: Manual Moderno; 1997. Spanish.

46. Carson C. The Effective Use of Effect Size Indices in Institutional Research. Available from: http://rlrw.bnu.edu.cn/NewsImage/ 2012410114358.pdf. Accessed November 8, 2015.

47. Fraser KD, O'Rourke HM, Wiens H, Lai J, Howell C, BrettMacLean PA. Scoping review of research on the arts, aging, and quality of life. Gerontologist. 2015;55(4):719-729.

48. Noice T, Noice H, Kramer AF. Participatory arts for older adults: a review of benefits and challenges. Gerontologist. 2014;54(5): 741-753.

49. Wurm S, Tesch-Römer C, Tomasik MJ. Longitudinal findings on agingrelated cognitions, control beliefs and health in later life. J Gerontol B Psychol Sci Soc Sci. 2007;62(3):156-164.

50. Levy BR, Slade MD, Kunkel SR, Klas SV. Longevity increased by positive self-perceptions of aging. J Pers Soc Psychol. 2002;83(3): 261-270.

51. Sniehotta FF, Schwarzer R, Scholz U, Schüz B. Action planning and coping planning for long-term lifestyle change: theory and assessment. Eur J Soc Psychol. 2005;35:565-576. 
52. Shilton T, Bauman A, Bull F, Sarmiento O. Effectiveness and challenges for promoting physical activity globally. In: McQueen DV, editor. Global Perspectives on Health Promotion Effectiveness. New York, NY: Springer; 2007:87-106.

53. Bandura A. Self-efficacy. In: Ramachaudran VS, editor. Encyclopedia of Human Behavior. Vol. 4. New York, NY: Academic Press; 1994:71-81.

54. Bandura A. Self-efficacy mechanism in human agency. Am Psychol. 1982;37:122-147.
55. Park DC. The basic mechanisms accounting for age-related decline in cognitive function. In: Park D, editor. Cognitive Aging: A Primer. New York, NY: Psychology Press; 2012:3-22.

56. Cavanaugh JC. Metamemory from a social-cognitive perspective. In: Park D, editor. Cognitive Aging: A Primer. New York, NY: Psychology Press; 2012:3-22.

57. Karanicolas PJ, Farrokhyar F, Bhandari M. Blinding: who, what, when, why, how? Can J Surg. 2010;53(5):345-348. 


\section{Supplementary materials}

Table SI Previous editions and outcomes of different versions of the Vital Aging program

\begin{tabular}{|c|c|c|c|c|c|c|c|c|}
\hline \multirow[t]{2}{*}{ Variables } & \multicolumn{3}{|c|}{ Study I: VA-M1,2 } & \multicolumn{3}{|c|}{ Study $2^{5,6}$} & \multicolumn{2}{|c|}{ Study $3^{3,4}$} \\
\hline & Residence & Community & C & VA-FF & VA-M & C & VA-M & C \\
\hline Active life & + & + & - & + & + & - & + & - \\
\hline Perceptions of aging & + & + & - & + & + & - & - & - \\
\hline Physical activity & - & + & - & - & + & - & + & - \\
\hline Nutritional habits & - & + & - & - & + & - & + & - \\
\hline Health status & - & - & - & - & - & - & - & - \\
\hline Social relationships & - & - & - & - & - & - & + & - \\
\hline Life satisfaction & - & + & - & - & + & - & - & - \\
\hline Subjective memory & & & & & & & + & - \\
\hline Mnemonic strategies & & & & & & & + & - \\
\hline Hedonic balance & & & & & & & + & - \\
\hline Negative emotions & & & & & & & + & - \\
\hline
\end{tabular}

Notes: + indicates improvements; - indicates no changes.

Abbreviations: VA-M, Vital Aging multimedia; VA-FF, Vital Aging face-to-face; C, Control group.

Regarding the multimedia version, it has been tested with consistent outcomes. In a first study, its efficacy was compared in different contexts, including community-dwelling older persons $(n=44)$, living in residential context $(n=13)$, and a control group $(n=31)$; results showed an increased frequency of cultural, intellectual, and social activities and a more positive view about aging in community and residential participants compared to those in the control group. ${ }^{1,2}$ Besides, those in community setting improved their nutrition habits, physical activity, and had better life satisfaction. Results remained in a 6-months follow-up with positive changes in health in the community group. No effects were reported in social relationships. A later study confirmed the impact of VA-M; it was implemented during 3 months. Seventy-three older adults participated in a 35-hour video lesson. Pretest and post-test were conducted comparing intervention and control groups. Results showed that participants in VA-M program improved in subjective health, general activities, frequency of physical exercise, and diet quality; in addition to improvements found in the previous editions, better subjective memory, use of mnemonic strategies, hedonic balance, frequency of social relationships, and fewer negative emotions (measures included only in this study) were reported., . $^{3,4}$

A separate study compares VA-FF $(n=28)$ and VA-M $(n=25)$ and a control group $(n=37)$ in community-dwelling older persons. Once again, improvements in the frequency of cultural, intellectual, and social activities and a more positive view about aging were reported. In addition, participants in the multimedia version reported more physical activity, better nutrition habits, and life satisfaction, compared to those in the face-to-face version. No effects were reported in social relationships and health. ${ }^{5,6}$

Regarding VA-eL, it was implemented between 2010 and 2012 through the learning management system, Learning Management System Moodle Platform. After several changes with the purpose to have an adapted cross-cultural program, it was launched at four participating universities: Autonomous University of Madrid, Catholic University of Chile, La Habana University, and the National Autonomous University of Mexico. Preliminary results obtained in the Spain subsample indicate that participants reported greater emotional balance and higher leisure and productive activities; the report has not been completed yet. ${ }^{7}$

\section{References}

1. Fernández-Ballesteros R, Caprara MG, García LF. Vivir con Vitalidad-M: un programa europeo multimedia [Vital aging-M: an European multimedia program]. Int Soc. 2004;13:63-85. Spanish.

2. Fernández-Ballesteros R, Caprara MG, Iñiguez J, García LF. Vital aging-M: an European multimedia programme. Psychol Spain. 2005; 9:1-12.

3. Fernández-Ballesteros R. Evaluation of "Vital Aging-M": a psychological program for promoting optimal aging. Eur Psychol. 2005;10(2): 146-156.

4. Caprara MG, Fernández-Ballesteros RF, Alessandri G. Promoting aging well: evaluation of Vital-Aging-Multimedia Program in Madrid, Spain. Health Promot Int. 2016;31(3):515-522.

5. Caprara MG. Envejecimiento con éxito: valoración de un programa [Successful aging: evaluation of a program] [PhD thesis]. Madrid: Autonomus University of Madrid; 2005.

6. Fernández-Ballesteros R, Caprara MG, Iñiguez J, García LF. Promoción del envejecimiento activo: efectos del programa «Vivir con vitalidad» ${ }^{\circledR}$ [Promoting active ageing: effects of the «Vital living» programme]. Rev Esp Geriatr Gerontol. 2005;40(2):92-102.

7. Caprara MG, Molina MA, Schettini R, et al. Active aging promotion: results from the Vital Aging Program. Curr Gerontol Geriatr Res. 2013; 2013:817813. 


\section{Publish your work in this journal}

Clinical Interventions in Aging is an international, peer-reviewed journal focusing on evidence-based reports on the value or lack thereof of treatments intended to prevent or delay the onset of maladaptive correlates of aging in human beings. This journal is indexed on PubMed Central, MedLine,

CAS, Scopus and the Elsevier Bibliographic databases. The manuscript management system is completely online and includes a very quick and fair peer-review system, which is all easy to use. Visit http://www.dovepress. com/testimonials.php to read real quotes from published authors. 